\title{
Regularization of electroweak monopole by charge screening and BPS energy bound
}

\author{
Pengming Zhang ${ }^{1, \mathrm{a}}$, Liping Zou ${ }^{2, \mathrm{~b}} \mathbb{\mathbb { C }}$, Y. M. Cho ${ }^{3,4, \mathrm{c}}$ \\ ${ }^{1}$ School of Physics and Astronomy, Sun Yat-Sen University, Zhuhai 519082, China \\ ${ }^{2}$ Institute of Modern Physics, Chinese Academy of Science, Lanzhou 730000, China \\ ${ }^{3}$ School of Physics and Astronomy, Seoul National University, Seoul 08826, Korea \\ ${ }^{4}$ Center for Quantum Spacetime, Sogang University, Seoul 04107, Korea
}

Received: 5 January 2020 / Accepted: 12 March 2020 / Published online: 27 March 2020

(C) The Author(s) 2020

\begin{abstract}
We show that the electroweak monopole can be regularized with a non-vacuum electromagnetic permittivity. This allows us to set a new BPS bound for the monopole mass, which implies that the mass may not be smaller than $2.98 \mathrm{TeV}$, more probably $3.75 \mathrm{TeV}$. We demonstrate that the same method can also regularize the Dirac monopole, which enhances the possibility to construct the Dirac monopole of mass of a few hundred $\mathrm{meV}$ in condensed matters. We discuss the physical implications of our result.
\end{abstract}

\section{Introduction}

Topology has played an essential role in physics. This is not accidental. All fundamental theories in physics are gauge theories which are described by the principal fiber bundle $[1,2]$. For example, the electrodynamics is described by the $\mathrm{U}(1)$ fiber bundle. And it has been well known that the principal fiber bundle has topological structure classified by the Chern classes. This tells that topology and physics can not be separated.

A best known example of such topological objects is the monopole. In 1931 Dirac has shown that, when the electromagnetic U(1) bundle becomes non-trivial, we can have the Dirac monopole in electrodynamics which obeys the famous charge quantization condition originating from the non-triviality of the U(1) bundle [3]. The Dirac monopole has been generalized to non-Abelian monopoles [4-10]. In particular it has been shown that in the unification of the weak and electromagnetic interactions the Dirac monopole trans-

\footnotetext{
a e-mail: zhangpm5@mail.sysu.edu.cn

b e-mail: zoulp@impcas.ac.cn (corresponding author)

c e-mail: ymcho0416@gmail.com
}

forms to the electroweak monopole of the standard model [11,12].

In the course of the electroweak unification the Dirac monopole changes it's character. First, it acquires the Wboson dressing and becomes a hybrid between the Dirac monopole and 'tHooft-Polyakov monopole. This is because the SU(2) part of the standard model naturally provides the $W$ boson dressing. Second, the magnetic charge becomes two times bigger. This is because the period of the electromagnetic $U(1)$ becomes $4 \pi$, not $2 \pi$, in this unification. Third, unlike the Dirac monopole which is optional, this one must exist if the standard model is correct. This is because the unification inevitably makes the electromagnetic U(1) nontrivial $[11,12]$.

This means that the discovery of this monopole, not the Higgs particle, should be interpreted as the final test (in fact the topological test) of the standard model. Moreover, this monopole could play important roles in cosmology [13]. In the early universe it could become the primordial black hole which could explain the dark matter, and become the seed of large scale structure of the universe. Most importantly, if discovered, this will become the first stable topological elementary particle in the history of physics.This makes the experimental detection of the electroweak monopole a most important issue after the discovery of the Higgs particle. For this reason the MoEDAL and ATLAS at LHC, the IceCube at the south pole, and similar detectors are actively searching for the monopole [14-20].

To detect the electroweak monopole, we have to know the mass. This is a most important piece of information needed to detect the monopole. There was no way to predict the mass of the Dirac monopole theoretically, which has made the search for the monopole a blind search in the dark room. The electroweak monopole known as the Cho-Maison monopole is a hybrid between Dirac and 'tHooft-Polyakov [11,12]. As 
such it has a Dirac-type point singularity which makes the energy divergent, and classically we can not calculate the mass of the electroweak monopole.

Fortunately we can estimate the mass. Intuitively the mass comes from exactly the same Higgs mechanism which makes the W-boson massive, except that here the coupling is magnetic. This implies that the monopole mass should be roughly $1 / \alpha$ times bigger than the $W$ boson mass, of the order of 10 $\mathrm{TeV}$. Moreover, one could argue that the quantum correction (the charge renormalization) might regularize the singularity and make the monopole energy finite. In fact we can obtain a finite energy electroweak monopole, replacing the hypercharge $\mathrm{U}(1)$ coupling by a running coupling, and show that the mass becomes around $7 \mathrm{TeV}[21,22]$.

But since the monopole mass is very important for us to detect the monopole a more precise estimate of the mass is needed. There have been two remarkable reports in this direction. It has been argued that, making the hypercharge running coupling more realistic, one could put a constraint on the upper limit on the mass to be around $5.57 \mathrm{TeV}$ $[23,24]$. Moreover, modifying the standard model slightly one could show that the mass has the Bogomol'nyi-PrasardSommerfeld (BPS) bound $2.37 \mathrm{TeV}$ [25].

The purpose of this paper is the following. First, we show that the singular Cho-Maison monopole can be regularized by the electromagnetic charge renormalization, the virtual electron-positron pair production, of the real electric charge. Second, we show that this regularization allows us to have a new BPS bound for the mass of the electroweak monopole given by $2.98 \mathrm{TeV}$, more realistically $3.75 \mathrm{TeV}$. Third, we demonstrate that the Dirac monopole can also be regularized by the same electric charge renormalization (i.e., the vacuum polarization).

These results are important for the following reasons. First, the regularization of the monopole with the electromagnetic permittivity assures that there is a realistic way to regularize the electroweak monopole. This enhances the possibility to find a finite energy electroweak monopole.

Second, the new BPS bound of the electroweak monopole mass is a very important information for the experiments searching for the electroweak monopole, in particular the MoEDAL and ATLAS experiments at LHC. This is because this implies that LHC may not be able to produce the monopole if the energy is less than $2.98 \mathrm{TeV}$.

Third, the regularization of Dirac monopole is very interesting from theoretical point of view, because as far as we know no method to regularize the Dirac monopole has been known so far. Moreover, from the practical point of view this enhances the possibility to find the Dirac monopole greatly, because this allows us to estimate mass of the Dirac monopole. Indeed, one of the reasons why the search for the Dirac monopole has not been successful is that it has been a blind search in the dark room, without any theoretical hint on the mass. Our regularization changes this situation.

The paper is organized as follows. In Sect. 2 we review the known electroweak monopole and dyon solutions. In Sect. 3 we show how the electromagnetic charge screening could regularize the singular Cho-Maison monopole and dyon. In Sect. 4 we construct the BPS electroweak monopole and set a new BPS bound for the monopole mass. In Sect. 5 we show that the same electromagnetic charge screening can regularize the Dirac monopole in electrodynamics and make the energy finite. Finally in the last section we discuss the physical implications of our results.

\section{Gauge independent abelianization of standard model}

It has widely been believed that the topological structure of the standard model is fundamentally different from the Georgi-Glashow model, so that it has no monopole while the other one allows the 'tHooft-Polyakov monopole. This is not true. To see this we start from the (bosonic sector of) Weinberg-Salam model,

$$
\begin{aligned}
\mathcal{L} & =-\left|\mathcal{D}_{\mu} \phi\right|^{2}-\frac{\lambda}{2}\left(|\phi|^{2}-\frac{\mu^{2}}{\lambda}\right)^{2}-\frac{1}{4} \mathbf{F}_{\mu \nu}^{2}-\frac{1}{4} G_{\mu \nu}^{2}, \\
\mathcal{D}_{\mu} \phi & =\left(\partial_{\mu}-i \frac{g}{2} \boldsymbol{\sigma} \cdot \mathbf{A}_{\mu}-i \frac{g^{\prime}}{2} B_{\mu}\right) \phi \\
& =D_{\mu} \phi-i \frac{g^{\prime}}{2} B_{\mu} \phi,
\end{aligned}
$$

where $\phi$ is the Higgs doublet, $\mathbf{A}_{\mu}, \mathbf{F}_{\mu \nu}$ and $G_{\mu \nu}, B_{\mu}$ are the gauge fields of the $\mathrm{SU}(2)$ and hypercharge $\mathrm{U}(1)$, and $D_{\mu}$ is the covariant derivative of $\mathrm{SU}(2)$.

Expressing $\phi$ with the Higgs field $\rho$ and unit doublet $\xi$ by

$\phi=\frac{\rho}{\sqrt{2}} \xi \quad\left(\xi^{\dagger} \xi=1\right)$,

we have

$$
\begin{aligned}
\mathcal{L}_{W S}= & -\frac{1}{2}\left(\partial_{\mu} \rho\right)^{2}-\frac{\rho^{2}}{2}\left|\mathcal{D}_{\mu} \xi\right|^{2}-\frac{\lambda}{8}\left(\rho^{2}-\rho_{0}^{2}\right)^{2} \\
& -\frac{1}{4} \mathbf{F}_{\mu \nu}^{2}-\frac{1}{4} G_{\mu \nu}^{2},
\end{aligned}
$$

where $\rho_{0}=\sqrt{2 \mu^{2} / \lambda}$ is the vacuum expectation value of the Higgs field. Notice that the hypercharge U(1) gauge interaction makes $\xi$ a $C P^{1}$ field [11]. which can naturally admit the $\pi_{2}\left(S^{2}\right)$ monopole topology.

To simplify this further we need the Abelian decomposition of the standard model. Start from the SU(2) gauge field $\mathbf{A}_{\mu}$ and let $\left(\hat{n}_{1}, \hat{n}_{2}, \hat{n}_{3}=\hat{n}\right)$ be an arbitrary right-handed orthonormal $S U(2)$ basis. Choose $\hat{n}$ to be the Abelian direction at each space-time point, and project out the restricted 
potential $\hat{A}_{\mu}$ imposing the isometry condition [26-29]

$$
\begin{aligned}
D_{\mu} \hat{n} & =0, \\
\mathbf{A}_{\mu} \rightarrow \hat{A}_{\mu} & =\tilde{A}_{\mu}+\tilde{C}_{\mu}, \\
\tilde{A}_{\mu} & =A_{\mu} \hat{n} \quad\left(A_{\mu}=\hat{n} \cdot \mathbf{A}_{\mu}\right), \quad \tilde{C}_{\mu}=-\frac{1}{g} \hat{n} \times \partial_{\mu} \hat{n} .
\end{aligned}
$$

The restricted potential has a dual structure, made of two potentials $\tilde{A}_{\mu}$ and $\tilde{C}_{\mu}$ which can also be described by two Abelian potentials $A_{\mu}$ and $C_{\mu}$,

$$
\begin{aligned}
\hat{F}_{\mu \nu} & =\partial_{\mu} \hat{A}_{\nu}-\partial_{\nu} \hat{A}_{\mu}+g \hat{A}_{\mu} \times \hat{A}_{\nu}=F_{\mu \nu}^{\prime} \hat{n}, \\
F_{\mu \nu}^{\prime} & =F_{\mu \nu}+H_{\mu \nu}=\partial_{\mu} A_{\nu}^{\prime}-\partial_{\nu} A_{\mu}^{\prime}, \\
F_{\mu \nu} & =\partial_{\mu} A_{\nu}-\partial_{\nu} A_{\mu}, \\
H_{\mu \nu} & =-\frac{1}{g} \hat{n} \cdot\left(\partial_{\mu} \hat{n} \times \partial_{\nu} \hat{n}\right)=\partial_{\mu} C_{\nu}-\partial_{\nu} C_{\mu}, \\
\hat{n} & =-\xi^{\dagger} \sigma \xi \\
C_{\mu} & =-\frac{2 i}{g} \xi^{\dagger} \partial_{\mu} \xi=-\frac{1}{g} \hat{n}_{1} \cdot \partial_{\mu} \hat{n}_{2}, \\
A_{\mu}^{\prime} & =A_{\mu}+C_{\mu} .
\end{aligned}
$$

Notice that the potential $C_{\mu}$ is determined uniquely up to the $U$ (1) gauge freedom which leaves $\hat{n}$ invariant. With

$\xi=\exp (-i \gamma)\left(\begin{array}{c}\sin \frac{\alpha}{2} \exp (-i \beta) \\ -\cos \frac{\alpha}{2}\end{array}\right)$,

we have

$\hat{n}=\left(\begin{array}{c}\sin \alpha \cos \beta \\ \sin \alpha \sin \beta \\ \cos \alpha\end{array}\right), \quad C_{\mu}=-\frac{1}{g}(1-\cos \alpha) \partial_{\mu} \beta$.

So when $\hat{n}=\hat{r}$, the potential $C_{\mu}$ describes the Dirac monopole and $\tilde{C}_{\mu}$ describes the Wu-Yang monopole $[4,9$, 10].

With this we obtain the gauge independent Abelian decomposition of the $S U(2)$ gauge field adding the gauge covariant valence part $\mathbf{W}_{\mu}$ which was excluded by the isometry [26-29]

$$
\begin{aligned}
\mathbf{A}_{\mu} & =\hat{A}_{\mu}+\mathbf{W}_{\mu}, \quad \mathbf{W}_{\mu}=W_{\mu}^{1} \hat{n}_{1}+W_{\mu}^{2} \hat{n}_{2}, \\
\mathbf{F}_{\mu \nu} & =\hat{F}_{\mu \nu}+\hat{D}_{\mu} \mathbf{W}_{\nu}-\hat{D}_{\nu} \mathbf{W}_{\mu}+g \mathbf{W}_{\mu} \times \mathbf{W}_{\nu}, \\
\hat{D}_{\mu} & =\partial_{\mu}+g \hat{A}_{\mu} .
\end{aligned}
$$

This is the gauge independent Abelian decomposition of the SU(2) gauge theory known as the Cho decomposition, ChoDuan-Ge (CDG) decomposition, or Cho-Faddeev-Niemi (CFN) decomposition [30-36]. Notice that once $\hat{n}$ is chosen, the decomposition follows automatically, regardless of the choice of gauge.

With this we can abelianize the Weinberg-Salam theory. Defining the electromagnetic field, $W$ boson, and $Z$ boson by

$$
\begin{aligned}
\left(\begin{array}{c}
A_{\mu}^{(\mathrm{em})} \\
Z_{\mu}
\end{array}\right) & =\frac{1}{\sqrt{g^{2}+g^{2}}}\left(\begin{array}{cc}
g & g^{\prime} \\
-g^{\prime} & g
\end{array}\right)\left(\begin{array}{l}
B_{\mu} \\
A_{\mu}^{\prime}
\end{array}\right) \\
& =\left(\begin{array}{cc}
\cos \theta_{\mathrm{w}} & \sin \theta_{\mathrm{w}} \\
-\sin \theta_{\mathrm{w}} & \cos \theta_{\mathrm{w}}
\end{array}\right)\left(\begin{array}{c}
B_{\mu} \\
A_{\mu}^{\prime}
\end{array}\right), \\
W_{\mu} & =\frac{1}{\sqrt{2}}\left(W_{\mu}^{1}+i W_{\mu}^{2}\right),
\end{aligned}
$$

we have

$$
\begin{aligned}
\left|D_{\mu} \xi\right|^{2} & =\frac{g^{2}}{4}\left(A_{\mu}^{\prime 2}+\mathbf{W}_{\mu}^{2}\right), \\
\left|\mathcal{D}_{\mu} \xi\right|^{2} & =\left|D_{\mu} \xi\right|^{2}+i g^{\prime} B_{\mu} \xi^{\dagger} D_{\mu} \xi+\frac{g^{\prime 2}}{4} B_{\mu}^{2} \\
& =\frac{1}{4} \mathbf{W}_{\mu}^{2}+\frac{g^{2}+g^{\prime 2}}{4} Z_{\mu}^{2},
\end{aligned}
$$

so that

$$
\begin{aligned}
\mathcal{L}= & -\frac{1}{2}\left(\partial_{\mu} \rho\right)^{2}-\frac{\lambda}{8}\left(\rho^{2}-\rho_{0}^{2}\right)^{2}-\frac{1}{4} F_{\mu \nu}^{\prime 2} \\
& -\frac{1}{4} G_{\mu \nu}^{2}-\frac{1}{2}\left|D_{\mu}^{\prime} W_{\nu}-D_{\nu}^{\prime} W_{\mu}\right|^{2} \\
& -\frac{g^{2}}{4} \rho^{2} W_{\mu}^{*} W_{\mu}-\frac{g^{2}+g^{2}}{8} \rho^{2} Z_{\mu}^{2} \\
& +i g F_{\mu \nu}^{\prime} W_{\mu}^{*} W_{\nu}+\frac{g^{2}}{4}\left(W_{\mu}^{*} W_{\nu}-W_{\nu}^{*} W_{\mu}\right)^{2} \\
= & -\frac{1}{2}\left(\partial_{\mu} \rho\right)^{2}-\frac{\lambda}{8}\left(\rho^{2}-\rho_{0}^{2}\right)^{2}-\frac{1}{4} F_{\mu \nu}^{(\mathrm{em})^{2}} \\
& \left.-\frac{1}{2} \mid\left(D_{\mu}^{(\mathrm{em})}+i e \frac{g}{g^{\prime}} Z_{\mu}\right) W_{\nu}-\left(D_{\nu}^{(\mathrm{em})}+i e \frac{g}{g^{\prime}} Z_{\nu}\right) W_{\mu}\right)\left.\right|^{2} \\
& -\frac{1}{4} Z_{\mu \nu}^{2}-\frac{g^{2}}{4} \rho^{2} W_{\mu}^{*} W_{\mu}-\frac{g^{2}+g^{\prime 2}}{8} \rho^{2} Z_{\mu}^{2} \\
& +i e\left(F_{\mu \nu}^{(\mathrm{em})}+\frac{g}{g^{\prime}} Z_{\mu \nu}\right) W_{\mu}^{*} W_{\nu}+\frac{g^{2}}{4}\left(W_{\mu}^{*} W_{\nu}-W_{\nu}^{*} W_{\mu}\right)^{2},
\end{aligned}
$$

where $D_{\mu}^{\prime}=\partial_{\mu}+i g A_{\mu}^{\prime}, D_{\mu}^{(\mathrm{em})}=\partial_{\mu}+i e A_{\mu}^{(\mathrm{em})}$ and $e$ is the electric charge

$e=\frac{g g^{\prime}}{\sqrt{g^{2}+g^{\prime 2}}}=g \sin \theta_{\mathrm{w}}=g^{\prime} \cos \theta_{\mathrm{w}}$.

We emphasize that this is not the Weinberg-Salam Lagrangian in the unitary gauge. This is a gauge independent expression.

The abelianization sheds a new light on the standard model. First of all, here the Higgs doublet disappears completely. Moreover, the $W$ boson and $Z$ boson acquire the mass when $\rho$ has the non-vanishing vacuum expectation value, without any "spontaneous" symmetry breaking by the Higgs doublet.

As importantly this clarifies the topological structure of the standard model. It has been asserted that the standard model has no monopole topology [37-40]. The basis of this 


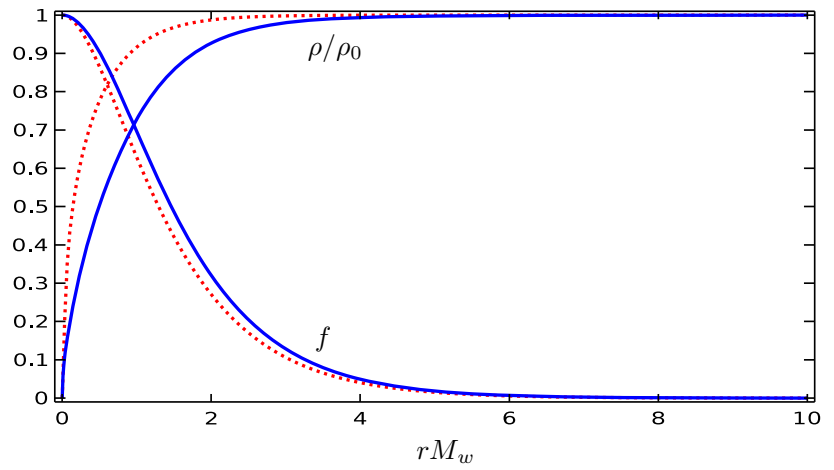

Fig. 1 The electroweak monopole solutions. The red curves represent the singular Cho-Maison monopole obtained by (15) and the blue curves represent the finite energy electroweak monopole obtained by (21) with $A=B=0$

"no-go theorem" is that, unlike the Higgs triplet in the GeorgiGlashow model, the Higgs doublet breaks the SU(2) symmetry completely. The above exercise, however, shows that this is wrong. First, the Higgs doublet disappears completely in this Abelianization. Second, in the absence of the weak bosons (11) reduces to electrodynamics, which admits Dirac monopole. More importantly, in this Abelianization the electromagnetic $\mathrm{U}(1)$ becomes non-trivial. This is evident from the fact that $A_{\mu}^{\prime}$ contains the monopole potential $C_{\mu}$. This confirms that, unlike in QED where the monopole becomes optional, the standard model must have the monopole.

In fact, this monopole topology can be traced back to the original Lagrangian (1). With the separation of the overall U(1) phase, (6) shows that $\xi$ becomes a $C P^{1}$ field which has the $S^{2}$ topology. Moreover, $\hat{n}$ defined by $\xi$ has the $S^{2}$ topology. So (1) has the $\pi_{2}\left(S^{2}\right)$ topology which describes the monopole.

The fact that the Lagrangian (11) is mathematically identical to (1) assures that it describes the standard model, at least at the classical level. But one might wonder if (11) describes the standard model at the quantum level. Logically it should, but this has not been demonstrated yet. It would be interesting to show that indeed at the quantum level they describe the same physics.

\section{Electroweak monopole: a review}

To construct the monopole we choose the ansatz in the spherical coordinates $(t, r, \theta, \varphi)$

$$
\begin{aligned}
\rho & =\rho(r), \quad \xi=i\left(\begin{array}{c}
\sin \frac{\theta}{2} e^{-i \varphi} \\
-\cos \frac{\theta}{2}
\end{array}\right), \\
\hat{n} & =-\xi^{\dagger} \boldsymbol{\sigma} \xi=\hat{r}, \quad C_{\mu}=-\frac{1}{g}(1-\cos \theta) \partial_{\mu} \varphi \\
\mathbf{A}_{\mu} & =\frac{1}{g} A(r) \partial_{\mu} t \hat{r}+\frac{1}{g}(f(r)-1) \hat{r} \times \partial_{\mu} \hat{r}, \\
B_{\mu} & =\frac{1}{g^{\prime}} B(r) \partial_{\mu} t-\frac{1}{g^{\prime}}(1-\cos \theta) \partial_{\mu} \varphi .
\end{aligned}
$$

Notice that the apparent string singularity along the negative $z$-axis in $\xi$ and $B_{\mu}$ is a pure gauge artefact which can easily be removed making $U(1)$ non-trivial. In terms of the physical fields the ansatz is expressed by

$$
\begin{aligned}
A_{\mu}^{(\mathrm{em})} & =e\left(\frac{A(r)}{g^{2}}+\frac{B(r)}{g^{\prime 2}}\right) \partial_{\mu} t-\frac{1}{e}(1-\cos \theta) \partial_{\mu} \varphi \\
W_{\mu} & =\frac{i}{g} \frac{f(r)}{\sqrt{2}} e^{i \varphi}\left(\partial_{\mu} \theta+i \sin \theta \partial_{\mu} \varphi\right), \\
Z_{\mu} & =\frac{e}{g g^{\prime}}(A(r)-B(r)) \partial_{\mu} t
\end{aligned}
$$

which assures that the ansatz is for a dyon.

With the ansatz we have the dyon equations of motion

$$
\begin{aligned}
& \ddot{\rho}+\frac{2}{r} \dot{\rho}-\frac{1}{2 r^{2}} f^{2} \rho=-\frac{1}{4}(B-A)^{2} \rho+\frac{\lambda}{2}\left(\rho^{2}-\rho_{0}^{2}\right) \rho, \\
& \ddot{f}-\frac{1}{r^{2}}\left(f^{2}-1\right) f=\left(\frac{g^{2}}{4} \rho^{2}-A^{2}\right) f, \\
& \ddot{A}+\frac{2}{r} \dot{A}-\frac{2}{r^{2}} f^{2} A=\frac{g^{2}}{4}(A-B) \rho^{2}, \\
& \ddot{B}+\frac{2}{r} \dot{B}=\frac{g^{\prime 2}}{4}(B-A) \rho^{2},
\end{aligned}
$$

which has the energy

$$
\begin{aligned}
E= & 4 \pi \int_{0}^{\infty} d r\left\{\frac{1}{2 g^{\prime 2} r^{2}}+\frac{1}{2}(r \dot{\rho})^{2}+\frac{\lambda}{8} r^{2}\left(\rho^{2}-\rho_{0}^{2}\right)^{2}\right. \\
& +\frac{1}{g^{2}}\left(\dot{f}^{2}+\frac{\left(f^{2}-1\right)^{2}}{2 r^{2}}+f^{2} A^{2}\right)+\frac{1}{4} f^{2} \rho^{2} \\
& \left.+\frac{r^{2}}{2}\left(\frac{\dot{A}^{2}}{g^{2}}+\frac{\dot{B}^{2}}{g^{\prime 2}}\right)+\frac{r^{2}}{8}(B-A)^{2} \rho^{2}\right\} .
\end{aligned}
$$

Obviously (15) has the singular monopole solution which describes the point monopole in Weinberg-Salam model

$$
\begin{aligned}
f & =0, \quad \rho=\rho_{0}, \quad A=B=0, \\
A_{\mu}^{(\mathrm{em})} & =-\frac{1}{e}(1-\cos \theta) \partial_{\mu} \varphi,
\end{aligned}
$$

which has the magnetic charge $4 \pi / e($ not $2 \pi / e)$.

Integrating (15) with $A=B=0$ and with the boundary condition

$\rho(0)=0, \quad f(0)=1, \quad \rho(\infty)=\rho_{0}, \quad f(\infty)=0$, 


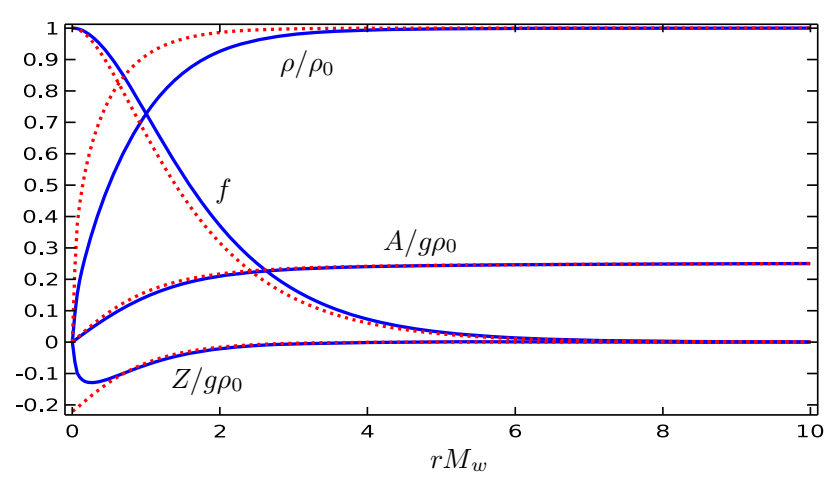

Fig. 2 The electroweak dyon solution. The red curves represent the singular Cho-Maison dyon and the blue curves represent the finite energy dyon obtained by (21). Here we have put $A_{0}=M_{W} / 2$ and $Z=A-B$

we obtain the singular (Cho-Maison) monopole solution dressed by the $W$ boson and Higgs field shown in Fig. 1 by the red curves. Moreover, with the boundary condition

$$
\begin{aligned}
\rho(0) & =0, \quad f(0)=1, \quad A(0)=0, \quad B(0)=b_{0}, \\
\rho(\infty) & =\rho_{0}, \quad f(\infty)=0, \quad A(\infty)=B(\infty)=A_{0},
\end{aligned}
$$

we can obtain the singular (Cho-Maison) dyon solution which has the extra electric charges $q_{e}=\left(4 \pi A_{1}\right) / e$. This is shown by the red curves in Fig. 2.

The electroweak monopole can be viewed as a hybrid between the Dirac monopole and the 'tHooft-Polyakov monopole, so that it has a topological U(1) point singularity at the center even though the $\mathrm{SU}(2)$ part is completely regular.

We can regularize the point singularity with the quantum correction at short distance, replacing the coupling constant $g^{\prime}$ of the hypercharge U(1) to an effective coupling which diverges at the origin [21,22]. To show this, we modify the Lagrangian (11) introducing a non-trivial hypercharge U(1) permittivity $\epsilon(\rho)$ which depends on $\rho$,

$$
\begin{aligned}
\mathcal{L}= & -\frac{1}{2}\left(\partial_{\mu} \rho\right)^{2}-\frac{\lambda}{8}\left(\rho^{2}-\rho_{0}^{2}\right)^{2}-\frac{1}{4}{F^{\prime}}_{\mu \nu}^{2} \\
& -\frac{1}{4} \epsilon(\rho) G_{\mu \nu}^{2}-\frac{1}{2}\left|D_{\mu}^{\prime} W_{\nu}-D_{\nu}^{\prime} W_{\mu}\right|^{2} \\
& -\frac{g^{2}}{4} \rho^{2} W_{\mu}^{*} W_{\mu}-\frac{1}{8} \rho^{2}\left(g A_{\mu}^{\prime}-g^{\prime} B_{\mu}\right)^{2} \\
& +i g F_{\mu \nu}^{\prime} W_{\mu}^{*} W_{\nu}+\frac{g^{2}}{4}\left(W_{\mu}^{*} W_{\nu}-W_{\nu}^{*} W_{\mu}\right)^{2} .
\end{aligned}
$$

The effective Lagrangian retains the $S U(2) \times U(1)$ gauge symmetry. Moreover, when $\epsilon$ approaches to one asymptotically, it reproduces the standard model. But $\epsilon$ effectively changes the $U(1)_{Y}$ gauge coupling $g^{\prime}$ to the "running" coupling $\bar{g}^{\prime}=g^{\prime} / \sqrt{\epsilon}$. This is because with the rescaling of $B_{\mu}$ to $B_{\mu} / g^{\prime}, g^{\prime}$ changes to $g^{\prime} / \sqrt{\epsilon}$. So, by making $\bar{g}^{\prime}$ infinite at the origin, we can regularize the Cho-Maison monopole $[21,22]$.
From this we have the modified dyon equation

$$
\begin{aligned}
\ddot{\rho}+\frac{2}{r} \dot{\rho}-\frac{f^{2}}{2 r^{2}} \rho= & \frac{\lambda}{2}\left(\rho^{2}-\rho_{0}^{2}\right) \rho-\frac{1}{4}(A-B)^{2} \rho \\
& +\frac{\epsilon^{\prime}}{2 g^{\prime 2}}\left(\frac{1}{r^{4}}-\dot{B}^{2}\right), \\
\ddot{f}-\frac{f^{2}-1}{r^{2}} f= & \left(\frac{g^{2}}{4} \rho^{2}-A^{2}\right) f, \\
\ddot{A}+\frac{2}{r} \dot{A}-\frac{2 f^{2}}{r^{2}} A= & \frac{g^{2}}{4} \rho^{2}(A-B), \\
\ddot{B}+2\left(\frac{1}{r}+\frac{\epsilon^{\prime}}{2 \epsilon} \dot{\rho}\right) \dot{B}= & -\frac{g^{\prime 2}}{4 \epsilon} \rho^{2}(A-B),
\end{aligned}
$$

where $\epsilon^{\prime}=d \epsilon / d \rho$. This has the energy

$$
\begin{aligned}
E= & 4 \pi \int_{0}^{\infty} d r\left\{\frac{\epsilon}{2 g^{\prime 2} r^{2}}+\frac{1}{2}(r \dot{\rho})^{2}+\frac{\lambda}{8} r^{2}\left(\rho^{2}-\rho_{0}^{2}\right)^{2}\right. \\
& +\frac{1}{g^{2}}\left(\dot{f}^{2}+\frac{\left(f^{2}-1\right)^{2}}{2 r^{2}}+f^{2} A^{2}\right)+\frac{1}{4} f^{2} \rho^{2} \\
& \left.+\frac{(r \dot{A})^{2}}{2 g^{2}}+\frac{\epsilon(r \dot{B})^{2}}{2 g^{\prime 2}}+\frac{r^{2}}{8}(A-B)^{2} \rho^{2}\right\} .
\end{aligned}
$$

To have a regular solution we let near the origin

$$
\begin{aligned}
\rho(r) & =r^{\delta}\left(h_{0}+h_{1} r+\cdots\right), \\
\bar{\epsilon} & =\left(\frac{\rho}{\rho_{0}}\right)^{n}\left[c_{0}+c_{1}\left(\frac{\rho}{\rho_{0}}\right)+\cdots\right],
\end{aligned}
$$

and find from the equation of motion that we need

$\delta=\frac{\sqrt{3}-1}{2}, \quad n>\frac{2}{\delta}=2(\sqrt{3}+1) \simeq 7.46$,

or

$\delta=\frac{2}{n-2}, \quad 2<n \leq 2(\sqrt{3}+1)$.

This assures that when $n>2$ we have finite energy monopole and dyon.

We can integrate (21) with $\epsilon=\left(\rho / \rho_{0}\right)^{n}$. The regularized monopole and dyon solutions with $n=6$ are shown in Figs. 1 and 2 by the blue curves. Notice that asymptotically the regularized solutions look very much like the singular solutions, except that for the finite energy dyon solution $Z$ becomes zero at the origin. With $n=6$ the monopole energy becomes

$E \simeq 0.65 \times \frac{4 \pi}{e^{2}} M_{W} \simeq 7.20 \mathrm{TeV}$.

This confirms that the ultraviolet regularization of the ChoMaison dyon is indeed possible.

Of course, we could choose different $\epsilon$ to obtain different solution. Indeed choosing the realistic $\epsilon$ which can fit the Higgs to two photon decay data better, one could argue that the mass of the electroweak monopole may not be larger than $5.57 \mathrm{TeV}[23,24]$. 


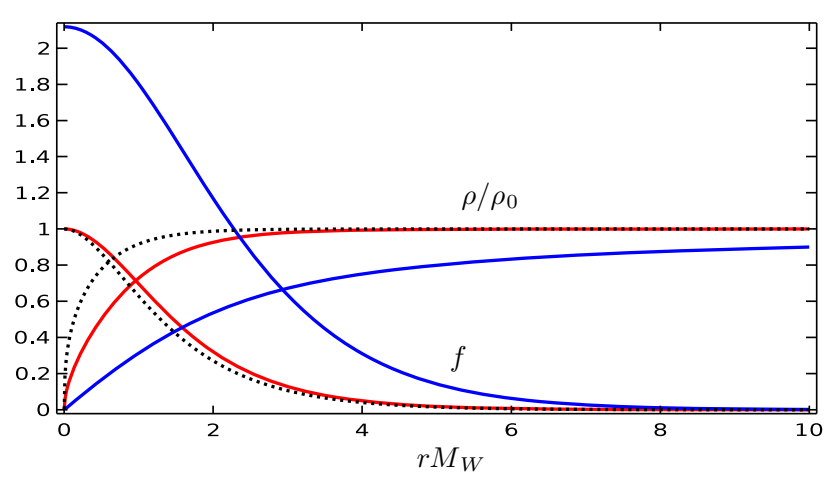

Fig. 3 The analytic electroweak monopole solution. The blue curves represent the analytic monopole given by (30) and the red curves represent the finite energy electroweak monopole obtained by (21). The Cho-Maison monopole is shown in dotted curves for comparison

One can have the analytic extension of the Cho-Maison monopole [21]. To see this notice that in the absence of the $Z$ boson (11) reduces to

$$
\begin{aligned}
\mathcal{L}= & -\frac{1}{2}\left(\partial_{\mu} \rho\right)^{2}-\frac{\lambda}{8}\left(\rho^{2}-\rho_{0}^{2}\right)^{2}-\frac{1}{4} F_{\mu \nu}^{(\mathrm{em})^{2}} \\
& -\frac{1}{2}\left|\left(D_{\mu}^{(\mathrm{em})} W_{\nu}-D_{\nu}^{(\mathrm{em})} W_{\mu}\right)\right|^{2}+i e F_{\mu \nu}^{(\mathrm{em})} W_{\mu}^{*} W_{\nu} \\
& -e^{2} \rho^{2} W_{\mu}^{*} W_{\mu}+\frac{e^{2}}{4}\left(W_{\mu}^{*} W_{\nu}-W_{\nu}^{*} W_{\mu}\right)^{2}+\delta \mathcal{L}, \\
\delta \mathcal{L}= & \left(e^{2}-\frac{g^{2}}{4}\right) \rho^{2} W_{\mu}^{*} W_{\mu}-\frac{e^{2}-g^{2}}{4}\left(W_{\mu}^{*} W_{\nu}-W_{\nu}^{*} W_{\mu}\right)^{2} .
\end{aligned}
$$

Notice that when $\delta \mathcal{L}=0$ this reduces to the Georgi-Glashow Lagrangian which describes the spontaneously broken SU(2) gauge theory [21].

With the monopole ansatz this has the energy density in the BPS limit (i.e., in the limit $\lambda$ vanishes)

$$
\begin{aligned}
E= & \frac{2 \pi}{g^{2}} \int_{0}^{\infty} d r\left\{g^{2}\left(r \dot{\rho}-\frac{1}{e r}\left(\frac{e^{2}}{g^{2}} f^{2}-1\right)\right)^{2}\right. \\
& +2(\dot{f}-e \rho f)^{2}+\frac{2 g^{2}}{e} \dot{\rho}\left(\frac{e^{2}}{g^{2}} f^{2}-1\right)+4 e \rho \dot{f} f \\
& \left.+\left(\frac{g^{2}}{2}-2 e^{2}\right) \frac{\rho^{2} f^{2}}{r^{2}}+\frac{g^{2}-e^{2}}{g^{2} r^{4}} f^{4}\right\}
\end{aligned}
$$

So, when the following BPS equation holds

$\dot{\rho}-\frac{1}{e r^{2}}\left(\frac{e^{2}}{g^{2}} f^{2}-1\right)=0, \quad \dot{f}-e \rho f=0$.

the monopole has the analytic solution [21]

$\rho=\rho_{0} \operatorname{coth}\left(e \rho_{0} r\right)-\frac{1}{e r}, \quad f=\frac{g \rho_{0} r}{\sinh \left(e \rho_{0} r\right)}$.

The BPS electroweak monopole is shown by the blue curves in Fig. 3. Notice that in this solution we have $f(0)=g / e$.
With (29) the energy has the bound

$$
\begin{gathered}
E \geq \frac{4 \pi}{g^{2}} \int_{0}^{\infty} d r\left\{\frac{g^{2}}{e^{2} r^{2}}\left(\frac{e^{2}}{g^{2}} f^{2}-1\right)^{2}+2 e^{2} \rho^{2} f^{2}\right. \\
\left.+\left(\frac{g^{2}}{4}-e^{2}\right) \frac{\rho^{2} f^{2}}{r^{2}}+\frac{e^{2}}{2 g^{\prime 2} r^{4}} f^{4}\right\}
\end{gathered}
$$

Notice that the last two terms come from $\delta \mathcal{L}$. So, if we neglect $\delta \mathcal{L}$ in the standard model, the model has the analytic solution similar to the Prasard-Sommerfeld solution in Georgi-Glashow model which could set the BPS bound for the monopole mass. Unfortunately the last term in (31) is divergent, so that the standard model as it is has no BPS bound monopole solution. As we will see, however, we can have the BPS electroweak monopole when we modify the standard model.

\section{Regularization of electroweak monopole with electromagnetic permittivity}

The above discussion shows that the quantum correction (the renormalization of the hypercharge coupling) could regularize the electroweak monopole and make the energy finite. Now we show that the renormalization of the real electric charge, can also regularize the Cho-Maison monopole.

This is because the point singularity of $B_{\mu}$ translates to the point singularity of $A_{\mu}^{(\mathrm{em})}$. To demonstrate this, consider the following effective Lagrangian of the standard model

$$
\begin{aligned}
\overline{\mathcal{L}}= & -\frac{1}{2}\left(\partial_{\mu} \rho\right)^{2}-\frac{\lambda}{8}\left(\rho^{2}-\rho_{0}^{2}\right)^{2}-\frac{1}{4} \bar{\epsilon}(\rho) F_{\mu \nu}^{(\mathrm{em})^{2}} \\
& \left.-\frac{1}{2} \mid\left(D_{\mu}^{(\mathrm{em})}+i e \frac{g}{g^{\prime}} Z_{\mu}\right) W_{\nu}-\left(D_{\nu}^{(\mathrm{em})}+i e \frac{g}{g^{\prime}} Z_{\nu}\right) W_{\mu}\right)\left.\right|^{2} \\
& -\frac{1}{4} Z_{\mu \nu}^{2}-\frac{g^{2}}{4} \rho^{2} W_{\mu}^{*} W_{\mu}-\frac{g^{2}+g^{\prime 2}}{8} \rho^{2} Z_{\mu}^{2} \\
& +i e\left(F_{\mu \nu}^{(\mathrm{em})}+\frac{g}{g^{\prime}} Z_{\mu \nu}\right) W_{\mu}^{*} W_{\nu}+\frac{g^{2}}{4}\left(W_{\mu}^{*} W_{\nu}-W_{\nu}^{*} W_{\mu}\right)^{2},
\end{aligned}
$$

where $\bar{\epsilon}$ is the real non-vacuum electromagnetic permittivity.

With the ansatz (14) we have the following dyon equations of motion

$$
\begin{aligned}
& \ddot{\rho}+\frac{2}{r} \dot{\rho}-\frac{f^{2}}{2 r^{2}} \rho=\frac{\lambda}{2}\left(\rho^{2}-\rho_{0}^{2}\right) \rho-\frac{1}{4}(B-A)^{2} \rho \\
& +\frac{\bar{\epsilon}^{\prime}}{2}\left(\frac{1}{e^{2} r^{4}}-e^{2}\left(\frac{\dot{A}}{g^{2}}+\frac{\dot{B}}{g^{\prime 2}}\right)^{2}\right), \\
& \ddot{f}-\frac{f^{2}-1}{r^{2}} f=\left(\frac{g^{2}}{4} \rho^{2}-A^{2}\right) f, \\
& \ddot{A}+\frac{2}{r} \dot{A}+e^{2} \frac{\bar{\epsilon}^{\prime}}{\bar{\epsilon}} \dot{\rho}\left(\frac{\dot{A}}{g^{2}}+\frac{\dot{B}}{g^{\prime 2}}\right)-\frac{2 e^{2}}{g^{2}}\left(\frac{g^{2}}{g^{\prime 2}}+\frac{1}{\bar{\epsilon}}\right) \frac{f^{2}}{r^{2}} A
\end{aligned}
$$




$$
\begin{aligned}
& =-\frac{g^{2}}{4} \rho^{2}(B-A), \\
\ddot{B} & +\frac{2}{r} \dot{B}+e^{2} \frac{\bar{\epsilon}^{\prime}}{\bar{\epsilon}} \dot{\rho}\left(\frac{\dot{A}}{g^{2}}+\frac{\dot{B}}{g^{\prime 2}}\right)+\frac{2 e^{2}}{g^{2}}\left(1-\frac{1}{\bar{\epsilon}}\right) \frac{f^{2}}{r^{2}} A \\
& =\frac{g^{\prime 2}}{4}(B-A) \rho^{2} .
\end{aligned}
$$

where $\bar{\epsilon}^{\prime}=d \bar{\epsilon} / d \rho$. To integrate it out and find a finite energy solution we have to choose a proper boundary condition.

To find a proper boundary condition notice that the Lagrangian gives us the following dyon energy

$$
\begin{aligned}
E= & 4 \pi \int_{0}^{\infty} d r\left\{\frac{\bar{\epsilon}}{2 e^{2} r^{2}}+\frac{1}{2}(r \dot{\rho})^{2}+\frac{\lambda r^{2}}{8}\left(\rho^{2}-\rho_{0}^{2}\right)^{2}\right. \\
& +\frac{1}{g^{2}}\left(\dot{f}^{2}+\frac{f^{2}\left(f^{2}-2\right)}{2 r^{2}}\right)+\frac{1}{4} f^{2} \rho^{2}+\frac{f^{2} A^{2}}{g^{2}} \\
& +\frac{r^{2}}{8}(A-B)^{2} \rho^{2}+\frac{r^{2}(\dot{A}-\dot{B})^{2}}{2\left(g^{2}+g^{\prime 2}\right)} \\
& \left.+\frac{\bar{\epsilon} e^{2} r^{2}}{2}\left(\frac{\dot{A}}{g^{2}}+\frac{\dot{B}}{g^{\prime 2}}\right)^{2}\right\} .
\end{aligned}
$$

This has two potentially divergent terms near the origin, the first and fifth terms. Assuming (23) we can make the first term finite. To make the fifth term finite we might require $f(0)=0$ or $f(0)=2$. But we find that this condition does not yield a finite energy solution.

A correct way to regularize the solution is to combine the two divergent terms and make it finite, since both have the same $O\left(r^{-2}\right)$ divergence at the origin. So, we let

$\bar{\epsilon}=\bar{\epsilon}_{0}+\bar{\epsilon}_{1}, \quad \bar{\epsilon}_{0}=\frac{g^{\prime 2}}{g^{2}+g^{\prime 2}}, \quad \bar{\epsilon}_{1} \simeq\left(\frac{\rho}{\rho_{0}}\right)^{n}$,

and find that the dyon energy is expressed by

$$
\begin{aligned}
E= & 4 \pi \int_{0}^{\infty} d r\left\{\frac{1}{2 e^{2} r^{2}}\left(\bar{\epsilon}_{0}\left(f^{2}-1\right)^{2}+\bar{\epsilon}_{1}\right)+\frac{1}{2}(r \dot{\rho})^{2}\right. \\
& +\frac{\lambda r^{2}}{8}\left(\rho^{2}-\rho_{0}^{2}\right)^{2}+\frac{1}{g^{2}} \dot{f}^{2}+\frac{1}{4} f^{2} \rho^{2}+\frac{f^{2} A^{2}}{g^{2}} \\
& +\frac{r^{2}}{8}(A-B)^{2} \rho^{2}+\frac{r^{2}(\dot{A}-\dot{B})^{2}}{2\left(g^{2}+g^{\prime 2}\right)} \\
& \left.+\frac{\bar{\epsilon} e^{2} r^{2}}{2}\left(\frac{\dot{A}}{g^{2}}+\frac{\dot{B}}{g^{\prime 2}}\right)^{2}\right\} .
\end{aligned}
$$

This tells that we can make the energy finite imposing the boundary condition $f(0)=1$. In other words we can regularize the monopole with the real electromagnetic permittivity $\bar{\epsilon}$ making $\bar{\epsilon}(0)$ finite, with the same boundary condition for $f$ as before. This is remarkable.

To see that this regularization works, consider the monopole solution first. In this case we can integrate (33) with $\bar{\epsilon}_{1}=\left(\rho / \rho_{0}\right)^{n}, A=B=0$, and with the boundary condition $f(0)=1$, and obtain the finite energy monopole

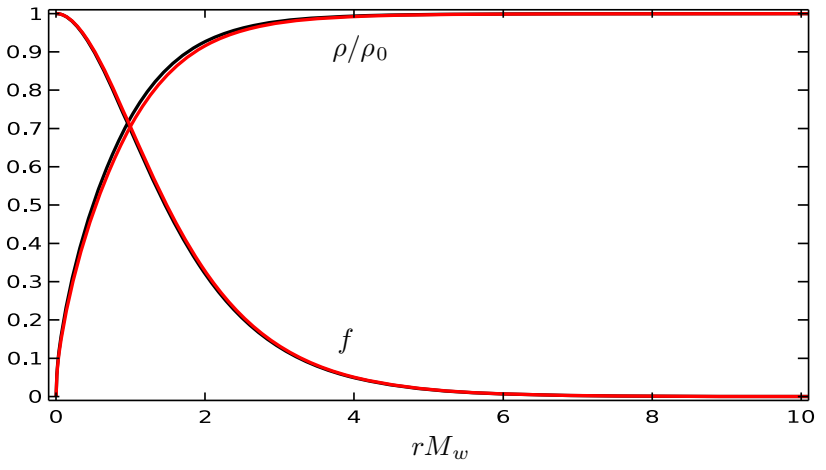

Fig. 4 The finite energy electroweak monopole solution regularized by the electromagnetic permittivity with $\mathrm{W}$ boson and Higgs scalar dressing. The red curve represents the regularized monopole solution with the non-trivial permittivity $\bar{\epsilon}_{1}=\left(\rho / \rho_{0}\right)^{6}$. For comparison we plot the monopole solution regularized by the hypercharge renormalization with the black curve (shown in Fig. 1)

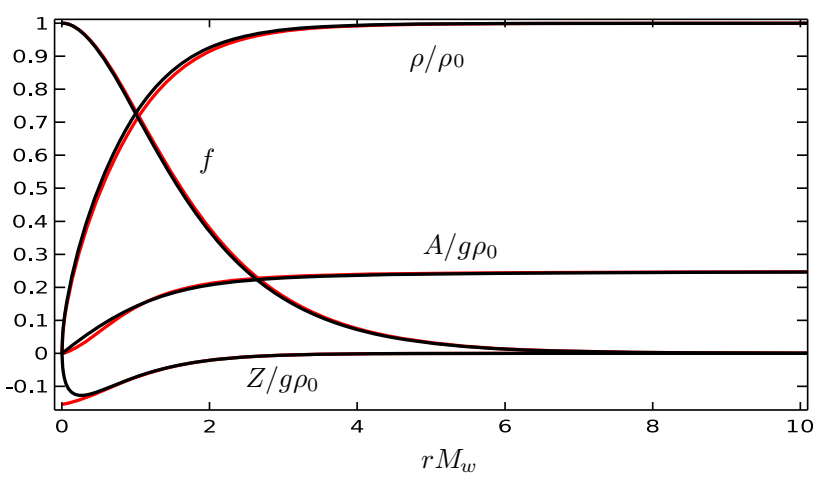

Fig. 5 The finite energy dyon solution (the red curves) regularized by the real electromagnetic permittivity $\bar{\epsilon}_{1}=\left(\rho / \rho_{0}\right)^{6}$ with the W boson, $\mathrm{Z}$ boson, and Higgs field dressing. For comparison we plot the finite energy dyon solution regularized by the hypercharge renormalization (shown in Fig. 2) by the black curves

solution (with $n>2$ ). The monopole solution with $n=6$ is shown in Fig. 4. We could generalize the monopole solution to dyon by solving (33). The solution with $n=6$ is shown in Fig. 5. Remarkably the solutions look almost identical to the solutions shown in Figs. 1 and 2. But the difference is that here $\bar{\epsilon}$ has a non-vanishing value $\bar{\epsilon}_{0}$ at the origin.

Of course, the energy depends on $n$, and we can plot the monopole energy in terms of $n$. This is shown in Fig. 6 in red dots. For $n=6$ the energy is given by $7.96 \mathrm{TeV}$. For comparison we plot the monopole energy regularized by $\epsilon$ (by the hypercharge renormalization) in blue dots. Notice that the energy approaches to the asymptotic value roughly given by $3.75 \mathrm{TeV}$ shown in black dotted line.

This confirms that we can indeed regularize the electroweak monopole and dyon solutions with the real electromagnetic permittivity. Moreover, this allows us to set a new bound for the mass of the electroweak monopole, as we will discuss in the following. 


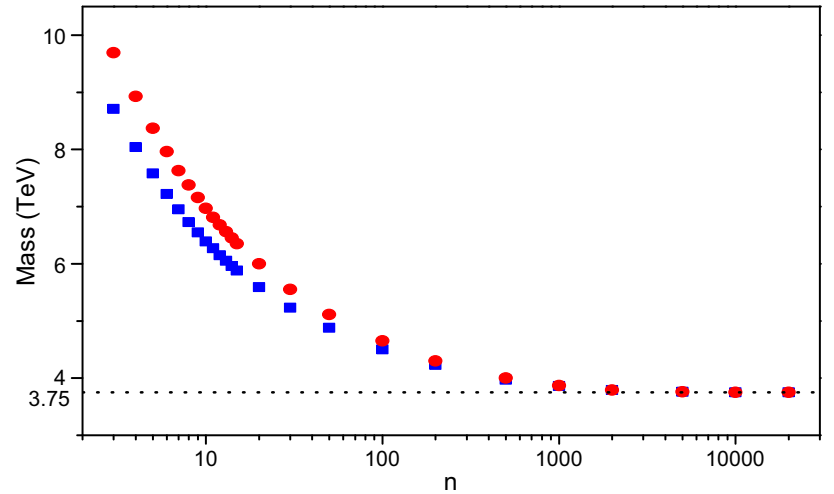

Fig. 6 The regularized monopole energy in terms of $n$ in log scale. The red dots represent the energy given by (36) with $A=B=0$. For comparison we plot the monopole energy given by (22) in blue dots. Here the dotted line represents the asymptotic energy when $n$ goes to infinity

\section{BPS electroweak monopoles}

We have shown that, if we replace the bare coupling of the standard model by the running coupling, we can have a finite energy monopole. This raises an interesting question. Can we set a BPS bound for the monopole with this type of modification? The BPS bound could be very useful for the experiments searching for the monopole. Indeed we can.

To show this we first consider the following modified Lagrangian [25]

$$
\begin{aligned}
\mathcal{L}= & -\frac{1}{2}\left(\partial_{\mu} \rho\right)^{2}-\frac{\lambda}{8}\left(\rho^{2}-\rho_{0}^{2}\right)^{2}-\frac{\epsilon}{4} G_{\mu \nu}^{2} \\
& -\left(\frac{\rho_{0}}{\rho}\right)^{2}\left\{\frac{1}{2}\left|D_{\mu}^{\prime} W_{\nu}-D_{\nu}^{\prime} W_{\mu}\right|^{2}-i g F_{\mu \nu}^{\prime} W_{\mu}^{*} W_{\nu}\right. \\
& \left.-\frac{g^{2}}{4}\left(W_{\mu}^{*} W_{\nu}-W_{\nu}^{*} W_{\mu}\right)^{2}\right\}-\frac{g^{2}}{4} \rho^{2} W_{\mu}^{*} W_{\mu} \\
& -\frac{1}{8} \rho^{2}\left(g A_{\mu}^{\prime}-g^{\prime} B_{\mu}\right)^{2} .
\end{aligned}
$$

Here $F_{\mu \nu}^{\prime}{ }^{2}$ term is missing, but this is acceptable because we are interested in the lower bound of monopole energy. Now, with the ansatz (14) the energy of the monopole in the BPS limit is given by

$$
\begin{aligned}
E= & 4 \pi \int_{0}^{\infty} d r\left\{\frac{\epsilon}{2 g^{\prime 2} r^{2}}+\frac{1}{2}(r \dot{\rho})^{2}+\left(\frac{\rho_{0}}{\rho}\right)^{2} \frac{\dot{f}^{2}}{g^{2}}+\frac{1}{4} \rho^{2} f^{2}\right\} \\
= & 4 \pi \int_{0}^{\infty} d r\left\{\frac{1}{2}\left(r \dot{\rho}-\frac{\sqrt{\epsilon}}{g^{\prime} r}\right)^{2}+\frac{1}{4}\left(\frac{2}{g} \frac{\rho_{0}}{\rho} \dot{f}+\rho f\right)^{2}\right. \\
& \left.+\frac{\sqrt{\epsilon}}{g^{\prime}} \dot{\rho}-\frac{\rho_{0}}{g} \dot{f} f\right\} \\
\geq & 4 \pi \int_{0}^{\infty} d r\left(\frac{\epsilon}{g^{\prime 2} r^{2}}+\frac{\rho^{2} f^{2}}{2}\right) .
\end{aligned}
$$

So, when monopole satisfies the Bogomol'nyi equation

$\dot{\rho}-\frac{\sqrt{\epsilon}}{g^{\prime} r^{2}}=0, \quad \dot{f}+\frac{g}{2} \frac{\rho}{\rho_{0}} \rho f=0$,

it has the Bogomol'nyi bound.

Integrating the first equation with $\epsilon=\left(\rho / \rho_{0}\right)^{n}$ and inserting the result to the the second equation, one can solve the Bogomol'nyi equation and obtain the following solution [25]

$$
\begin{aligned}
\rho(r)= & \rho_{0}\left(1+\frac{n-2}{2 g^{\prime} \rho_{0} r}\right)^{-\frac{2}{n-2}}, \\
f(r)= & f(0) \exp \left[-\frac{g(n-2) \rho_{0} r}{2(n+2)}\left(\frac{4 g^{\prime} \rho_{0} r}{n-2}\right)^{\frac{4}{n-2}}\right. \\
& \left.\times{ }_{2} F_{1}\left(1+\frac{4}{n-2}, \frac{4}{n-2} ; 2+\frac{4}{n-2} ;-\frac{4 g^{\prime} \rho_{0} r}{n-2}\right)\right],
\end{aligned}
$$

where ${ }_{2} F_{1}$ is the hypergeometric function. The solution with $n=6$ and $f(0)=1$ is shown in Fig. 7 in black curves.

It has been shown that, if one neglects the $\epsilon$ term in (38) one has the BPS energy bound given by [25]

$E \geq \frac{2 \pi}{g} \rho_{0}=\frac{4 \pi}{e^{2}} \sin ^{2} \theta_{\mathrm{w}} M_{\mathrm{w}} \simeq 2.37 \mathrm{TeV}$.

One might try to obtain a better bound including the $\epsilon$ term. With $\epsilon=\left(\rho / \rho_{0}\right)^{n}$ we have from (39)

$$
\begin{aligned}
E & \geq 4 \pi \int_{0}^{\infty} \frac{\rho_{0}}{g} f \dot{f} d r+\frac{4 \pi}{g^{\prime}} \int_{0}^{\infty}\left(\frac{\rho}{\rho_{0}}\right)^{n / 2} d \rho \\
& =4 \pi\left(\frac{1}{2 g}+\frac{2}{n+2} \frac{1}{g^{\prime}}\right) \rho_{0} \\
& =\frac{4 \pi}{e^{2}} \sin ^{2} \theta_{\mathrm{w}}\left(1+\frac{4}{n+2} \cot \theta_{\mathrm{w}}\right) M_{\mathrm{w}} .
\end{aligned}
$$

But this bound approaches to the above bound as $n$ goes to infinity.

Now, we discuss a more realistic way to set the BPS bound for the electroweak monopole. Consider the Lagrangian (32) modified by the real electromagnetic permittivity. Compared with the Lagrangian (37), this has a "minimum" modification of the standard model because (32) becomes the WeinbergSalam Lagrangian (1) with $\bar{\epsilon}=1$. This means that, if (32) has the BPS limit, the solution becomes more reliable than the BPS solution given by (37).

To obtain the BPS monopole solution from (32) notice that, with $A=B=0$ the monopole energy (36) in the BPS limit can be expressed by

$$
\begin{aligned}
E= & 4 \pi \int_{0}^{\infty} d r\left\{\frac{\bar{\epsilon}_{1}}{2 e^{2} r^{2}}+\frac{1}{2}(r \dot{\rho})^{2}\right. \\
& \left.+\frac{1}{2 g^{2} r^{2}}\left(f^{2}-1\right)^{2}+\frac{1}{g^{2}} \dot{f}^{2}+\frac{1}{4} \rho^{2} f^{2}\right\}
\end{aligned}
$$




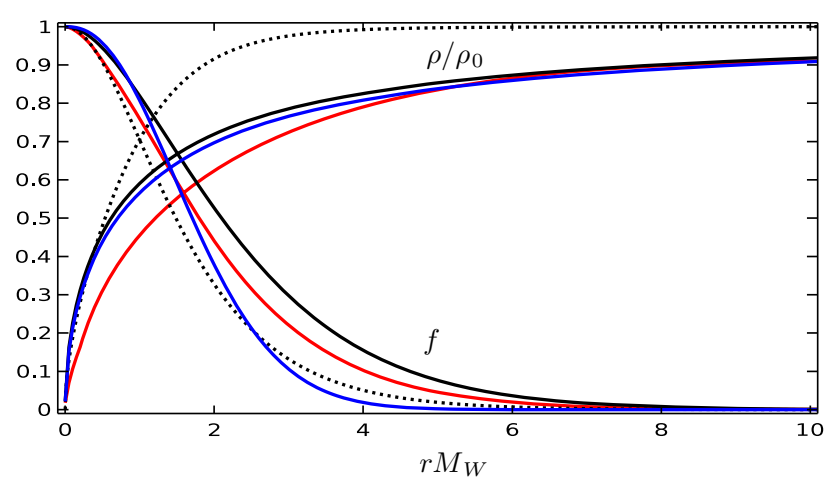

Fig. 7 The BPS electroweak monopole solutions. The black curves represent the solution given by (40), the blue curves represent the solution given by (44), and the red curves represent the solution given by (50). The monopole solution regularized by the real electromagnetic permittivity given by (33) is shown in dotted curves for comparison

$$
\begin{aligned}
= & 4 \pi \int_{0}^{\infty} d r\left\{\frac{1}{2}\left(r \dot{\rho}-\frac{\sqrt{\bar{\epsilon}_{1}}}{e r}\right)^{2}+\frac{\sqrt{\bar{\epsilon}_{1}}}{e} \dot{\rho}\right. \\
& \left.+\left(\frac{\dot{f}}{g}+\frac{1}{2} \rho f\right)^{2}-\frac{1}{g} \rho f \dot{f}+\frac{1}{2 g^{2} r^{2}}\left(f^{2}-1\right)^{2}\right\} .
\end{aligned}
$$

So when we have the Bogomol'nyi equation

$\dot{\rho}-\frac{\sqrt{\bar{\epsilon}_{1}}}{e r^{2}}=0, \quad \dot{f}+\frac{g}{2} \rho f=0$,

we have the BPS energy bound

$E \geq 4 \pi \int_{0}^{\infty} d r\left\{\frac{\bar{\epsilon}_{1}}{e^{2} r^{2}}+\frac{1}{2} \rho^{2} f^{2}+\frac{1}{2 g^{2} r^{2}}\left(f^{2}-1\right)^{2}\right\}$.

This shows that, with (35) the energy has the BPS bound when $f(0)=1$. Clearly this bound is better than (38).

Integrating the BPS equation with $f(0)=1$ and $n=6$, we obtain the monopole solution shown in Fig. 7 in blue curves. This has the minimum energy given by $6.26 \mathrm{TeV}$. But this bound has a drawback that it depends on the form of $\bar{\epsilon}_{1}$.

To have a new bound which is independent of $\bar{\epsilon}_{1}$, notice that when $n$ goes to infinity, $\bar{\epsilon}_{1}$ approaches to zero, so that we can neglect it. In this limit, we have

$\rho=\rho_{0}, \quad f=\exp \left(-\frac{g}{2} \rho_{0} r\right)$.

from (44). Moreover, (45) can be expressed by

$$
\begin{aligned}
E & >4 \pi \int_{0}^{\infty} d r\left\{\frac{\rho_{0}^{2}}{2} \exp \left(-g \rho_{0} r\right)\right. \\
& \left.+\frac{1}{2 g^{2} r^{2}}\left(\exp \left(-g \rho_{0} r\right)-1\right)^{2}\right\} \\
& \simeq 5.89 \mathrm{TeV} .
\end{aligned}
$$

This is interesting. But this result may not be so reliable for the following reasons. First, with $\rho=\rho_{0}$ the first term in
(45) diverges. Second, in the limit $n$ goes to infinity, we must have $\rho=0$ for any finite value of $r$.

Interestingly (32) allows another BPS bound. To see this notice that the monopole energy (48) can also be expressed by

$$
\begin{aligned}
E= & 4 \pi \int_{0}^{\infty} d r\left\{\frac{\bar{\epsilon}_{1}}{2 e^{2} r^{2}}+\frac{1}{2}\left(r \dot{\rho}+\frac{1}{g r}\left(f^{2}-1\right)\right)^{2}\right. \\
& \left.+\frac{1}{g^{2}}\left(\dot{f}+\frac{g}{2} \rho f\right)^{2}+\frac{1}{g} \dot{\rho}\left(f^{2}-1\right)-\frac{1}{g} \rho f \dot{f}\right\} .
\end{aligned}
$$

So, the energy has the following BPS bound

$$
E \geq 4 \pi \int_{0}^{\infty} d r\left\{\frac{\bar{\epsilon}_{1}}{2 e^{2} r^{2}}+\frac{1}{2} \rho^{2} f^{2}+\frac{1}{g^{2} r^{2}}\left(f^{2}-1\right)^{2}\right\},
$$

when we have the following BPS equation

$\dot{\rho}+\frac{1}{g r^{2}}\left(f^{2}-1\right)=0, \quad \dot{f}+\frac{g}{2} \rho f=0$.

This has two interesting features. First, this BPS equation is independent of $\bar{\epsilon}_{1}$. Second, it looks remarkably similar to the Prasard-Sommerfeld equation in Georgi-Glashow model. The only difference is the factor $1 / 2$ in the second equation. Integrating (50) with $n=6$ we obtain the BPS monopole solution shown in Fig. 7 in red curves, which has the energy $3.96 \mathrm{TeV}$.

In the limit $n$ goes to infinity, we can neglect the first term in (49) and set a $\bar{\epsilon}_{1}$ independent BPS bound

$$
\begin{aligned}
E & >4 \pi \int_{0}^{\infty} d r\left\{\frac{1}{2} \rho^{2} f^{2}+\frac{1}{g^{2} r^{2}}\left(f^{2}-1\right)^{2}\right\} \\
& =0.269 \times \frac{4 \pi}{e^{2}} M_{\mathrm{w}} \simeq 2.98 \mathrm{TeV} .
\end{aligned}
$$

This bound provides a new BPS bound for the electroweak monopole mass based on (32), which improves the existing bound (41). The result strongly implies that the monopole mass may not be smaller than $2.98 \mathrm{TeV}$.

This bound should be compared to the bound $3.75 \mathrm{TeV}$ shown in Fig. 6. The difference comes (at least partly) from the fact that here we have assumed $\lambda=0$ and neglected Higgs potential energy. Another difference is that the bound given by Fig. 6 is obtained using the full equation of motion, not the BPS equation. In this sense the bound $3.75 \mathrm{TeV}$ could be viewed more realistic, although this is obtained numerically with $\bar{\epsilon}_{1}=\left(\rho / \rho_{0}\right)^{n}$.

So far we have considered the electroweak monopole dressed by both $\mathrm{W}$ boson and Higgs field. Now, one might wonder if we can have a regularized monopole solution without the $W$ boson (i.e., without $f$ ), with only the Higgs field. At first glance this appears to be a meaningless question, because the $\mathrm{W}$ boson is an essential ingredient of the standard model. Moreover, (15) tells that, without $f$ Higgs field 
does not couple to the monopole. But this becomes a relevant question for the following reasons.

First, when we introduce the quantum correction, the Higgs field does couple to the monopole. This is clear from (21) and (33). So mathematically this becomes an interesting question. More importantly, from the physical point of view this becomes an important issue because this translates to the regularization of Dirac monopole.

It is well known that a major difficulty to find the Dirac monopole experimentally has been that there was no theoretical estimate of the mass, so that people had no idea where to look at the monopole. Now, it must be clear that in the absence of the $W$ boson the electroweak monopole looks very much like the Dirac monopole. So, if we could regularize the electroweak monopole in the absence of the $W$ boson, we might apply this regularization to estimate the mass of the Dirac monopole. This makes the regularization of the electroweak monopole in the absence of the $W$ boson a very interesting problem.

To find the electroweak monopole solution in the absence of the $\mathrm{W}$ boson, consider (21). In this case we can integrate (21) and find the the finite energy monopole solution which has the Higgs field dressing. This is shown in Fig. 8 in red curve.

Similarly, we can find the monopole solution regularized by the real electric permittivity integrating (33). In this case, we have to choose $\bar{\epsilon}_{0}=0$ to make the energy (36) finite. The Higgs field dressing of the regularized monopole solution shown in Fig. 8 in blue curve. Remarkably the solution is almost identical to the red curve regularized by $\epsilon$. From (34) we find the energy of this monopole for $n=6$ to be around

$E \simeq 0.46 \times \frac{4 \pi}{e^{2}} M_{\mathrm{w}} \simeq 5.09 \mathrm{TeV}$.

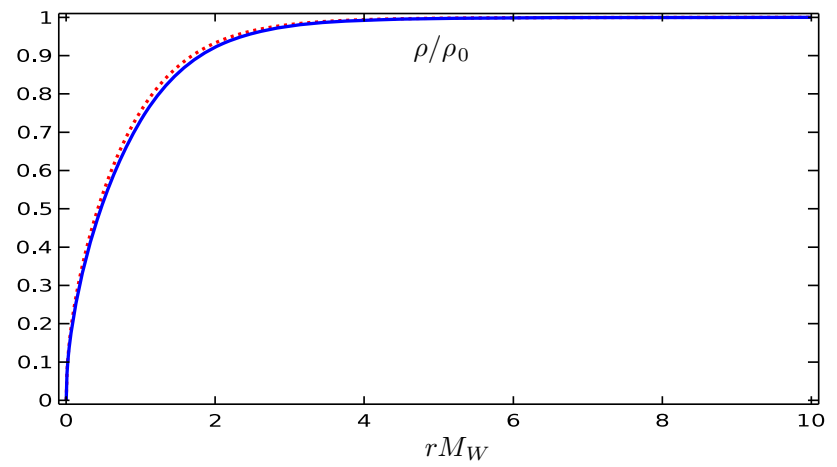

Fig. 8 The Higgs field dressings of the regularized monopole in the absence of the $\mathrm{W}$ boson. The blue curve corresponds to the real electromagnetic permittivity $\bar{\epsilon}=\left(\rho / \rho_{0}\right)^{6}$ and the red curve corresponds to the hypercharge permittivity $\tilde{\epsilon}=\left(\rho / \rho_{0}\right)^{6}$. Exactly the same solution also describes the finite energy Dirac monopole regularized by the vacuum polarization
This shows that we can indeed regularize the electroweak monopole without the $W$ boson with the real electromagnetic permittivity which describes the electric charge screening. One may wonder if we can generalize the above monopole solution to a dyon, integrating (33) with $f=0$. But we find that there is no solution which has non-trivial $A$ and $B$.

\section{Regularization of dirac monopole}

Since the standard model, in the absence of the W-boson reduces to the electrodynamics, we may apply the above regularization of the electroweak monopole to regularize the Dirac monopole. To show this we start from the following electromagnetic $\mathrm{U}(1)$ gauge theory coupled to the neutral scalar field $\rho$,

$\mathcal{L}=-\frac{1}{2}\left(\partial_{\mu} \rho\right)^{2}-\frac{\lambda}{8}\left(\rho^{2}-\rho_{0}^{2}\right)^{2}-\frac{1}{4} \bar{\epsilon}(\rho) F_{\mu \nu}^{2}$.

Here $F_{\mu \nu}$ is the real electromagnetic field and $\rho$ is an emergent scalar field which represents the density of electronpositron pairs responsible for the charge screening. Obviously this Lagrangian is mathematically identical to the Lagrangian (27) in the absence of the $W$ and $Z$ bosons. But from the physical point of view we emphasize that this Lagrangian is completely independent of the standard model. In particular, here $\rho$ is not the Higgs field which makes the photon massive, so that $\rho_{0}$ does not represent the vacuum expectation value of the Higgs field in the standard model.

Now, choose the following ansatz for the Dirac monopole

$$
\begin{aligned}
\rho & =\rho(r), \\
A_{\mu} & =A(r) \partial_{\mu} t-\frac{1}{2 e}(1-\cos \theta) \partial_{\mu} \varphi .
\end{aligned}
$$

With this we have the monopole equation of motion

$$
\begin{aligned}
& \ddot{\rho}+\frac{2}{r} \dot{\rho}=\frac{\lambda}{2}\left(\rho^{2}-\rho_{0}^{2}\right) \rho+\frac{\bar{\epsilon}^{\prime}}{2 e^{2}}\left(\frac{1}{r^{4}}-\dot{A}^{2}\right), \\
& \ddot{A}+\left(\frac{2}{r}+\frac{\bar{\epsilon}^{\prime}}{\bar{\epsilon}} \dot{\rho}\right) \dot{A}=0 .
\end{aligned}
$$

which becomes exactly identical to (33) when $f=0$ and $A=B$. This means that mathematically (33) in the absence of $W$ and $Z$ bosons describes the monopole described by (55).

This means that mathematically the monopole solution identical to the one shown in Fig. 8 can describe the regularized Dirac monopole dressed by the Higgs field in QED. But from the physical point of view this monopole is completely different. In particular here the monopole energy is 
expressed by

$E=4 \pi \int_{0}^{\infty} d r\left\{\frac{\bar{\epsilon}}{2 e^{2} r^{2}}+\frac{1}{2}(r \dot{\rho})^{2}+\frac{\lambda r^{2}}{8}\left(\rho^{2}-\rho_{0}^{2}\right)^{2}\right\}$,

which, in the limit $\lambda$ goes to zero, is given by

$E \simeq 0.25 \times \frac{4 \pi}{e} \rho_{0}$,

where $\rho_{0}$ now is in principle arbitrary, not related to the $W$ boson mass.

The result in this section is unexpected, because it confirms that we can regularize not only the electroweak monopole but also the Dirac monopole, replacing the vacuum electromagnetic permittivity with a real electromagnetic permittivity. As far as we understand there has been no mechanism which makes the energy of Dirac monopole finite. Our result shows that the electric permittivity in real matters could regularize the Dirac monopole.

It must be emphasized that the regularization of Dirac monopole is independent of the regularization of the ChoMaison monopole in the standard model. In particular, $\rho_{0}$ in (53) is arbitrary (not related to the vacuum expectation value of Higgs field in the standard model, so that the mass of the Dirac monopole given by (57) is completely independent of the mass of the electroweak monopole. Indeed, in condensed matters $\rho_{0}$ could easily be of the order of $\mathrm{meV}$, the vacuum expectation value of the Higgs field in Landau-Ginzburg theory. This implies that a regularized Dirac monopole of mass around hundred meV could exist in real condensed matters.

\section{Conclusion}

The fact that the energy of the Cho-Maison monopole is infinite has made some people to doubt the existence of the monopole in the standard model. But we emphasize that the existence of the monopole is determined by the topology of the standard model, not the classical energy of the monopole $[11,12]$. Moreover, since the standard model has the monopole topology, the electroweak monopole must exist.

For this reason the search for the electroweak monopole has been taken seriously [14-20]. A crucial piece of information needed to make the search successful is the monopole mass. Generally speaking, the mass is expected to be around 4-10 TeV [21,23-25], but we need a more precise prediction.

In this paper we showed that indeed the electromagnetic permittivity can regularize the electroweak monopole. This has deep implications. First of all, this shows that there is a more natural way to regularize the monopole, which makes the electroweak monopole more realistic. Perhaps more importantly, this sets a new limit on the monopole mass. Indeed, our result strongly implies that the mass of the monopole may not be smaller than $3 \mathrm{TeV}$ (more realistically $3.75 \mathrm{TeV}$ ). This could be an important information for experiments to detect the electroweak monopole, in particular the MoEDAL and ATLAS experiments at CERN, because the present $14 \mathrm{TeV}$ LHC could produce the monopole only when the monopole mass is less than $7 \mathrm{TeV}$ [14-18].

Furthermore, our result shows that the charge screening could also regularize the Dirac monopole. This could revitalize the search for the Dirac monopole. One reason why the experimental search for the Dirac monopole has not been so successful so far is that it has been a blind search in the dark room, without any theoretical hint on the mass [41]. But a more serious reason is that the Dirac monopole may not exist in nature as a fundamental particle, because the electroweak unification transforms it to the electroweak monopole.

But there is no reason why this monopole could not exist as a quasi-stable particle. To understand this point, consider the well known Abrikosov vortex in ordinary superconductors $[42,43]$. Although this vortex does not exist as a fundamental object, we can create it in condensed matters applying the magnetic field from the outside. Exactly the same way, we could create the Dirac monopole in condensed matters by brute force, applying a spherically symmetric magnetic field. Certainly this would not be easy, but there is no reason why this could not be done.

This strongly implies that for the Dirac monopole what is important is creating it in condensed matters, rather than searching for the existing one in nature. Our analysis shows that in this case the mass of the Dirac monopole could be of the order of $100 \mathrm{meVs}$, since $\rho_{0}$ in condensed matters is expected to be of the order of meV. This is remarkable.

Acknowledgements The work is supported in part by the National Research Foundation of Korea funded by the Ministry of Education (Grants 2015-R1D1A1A0-1057578, 2018-R1D1A1B0-7045163), National Natural Science Foundation of China (Grant 11975320 and Grant 11805242), and by Center for Quantum Spacetime, Sogang University, Korea.

Data Availability Statement This manuscript has no associated data or the data will not be deposited. [Authors' comment: This is a theoretical paper and therefore has no data to deposit.]

Open Access This article is licensed under a Creative Commons Attribution 4.0 International License, which permits use, sharing, adaptation, distribution and reproduction in any medium or format, as long as you give appropriate credit to the original author(s) and the source, provide a link to the Creative Commons licence, and indicate if changes were made. The images or other third party material in this article are included in the article's Creative Commons licence, unless indicated otherwise in a credit line to the material. If material is not included in the article's Creative Commons licence and your intended use is not permitted by statutory regulation or exceeds the permitted use, you will need to obtain permission directly from the copyright holder. To view a copy of this licence, visit http://creativecomm ons.org/licenses/by/4.0/.

Funded by $\mathrm{SCOAP}^{3}$. 


\section{References}

1. Y.M. Cho, J. Math. Phys. 16, 2029 (1975)

2. Y.M. Cho, P.G.O. Freund, Phys. Rev. D 12, 1711 (1975)

3. P.A.M. Dirac, Phys. Rev. 74, 817 (1948)

4. T.T. Wu, C.N. Yang, Phys. Rev. D 12, 3845 (1975)

5. G. 'tHooft, Nucl. Phys. B 79, 276 (1974)

6. A. Polyakov, JETP Lett. 20, 194 (1974)

7. M.K. Prasad, C.M. Sommerfield, Phys. Rev. Lett. 35, 760 (1975)

8. C.P. Dokos, T.N. Tomaras, Phys. Rev. D 21, 2940 (1980)

9. Y.M. Cho, Phys. Rev. Lett. 44, 1115 (1980)

10. Y.M. Cho, Phys. Lett. B 115, 125 (1982)

11. Y.M. Cho, D. Maison, Phys. Lett. B 391, 360 (1997)

12. Y. Yang, Proc. Roy. Soc. A 454, 155 (1998). See also Yisong Yang, Solitons in Field Theory and Nonlinear Analysis (Springer Monographs in Mathematics), (Springer-Verlag) 2001

13. Y.M. Cho, Philos. Trans. A 377, 20190038 (2019)

14. B. Acharya et al. (MoEDAL Collaboration), Phys. Rev. Lett. 118, 061801 (2017)

15. B. Acharya et al. (MoEDAL Collaboration), Phys. Rev. Lett. 123, 021802 (2019)

16. ATLAS Collaboration, Phys. Rev. Lett. 109, 261803 (2012)

17. ATLAS Collaboration, Euro. Phys. J. C 75, 362 (2015)

18. ATLAS Collaboration, Phys. Rev. Lett. (in press)

19. R. Abbasi et al. (IceCube Collaboration) Phys. Rev. D 87, 022001 (2013)

20. M. Ahlers, K. Helbing, C. Heros, Eur. Phys. J. C 78, 924 (2018)

21. Kyoungtae Kimm, J.H. Yoon, Y.M. Cho, Eur. Phys. J. C 75, 67 (2015)
22. Kyoungtae Kimm, J.H. Yoon, S.H. Oh, Y.M. Cho, Mod. Phys. Lett. A 31, 1650053 (2016)

23. J. Ellis, N.E. Mavromatos, T. You, Phys. Lett. B 756, 29 (2016)

24. J. Alexandre, N.E. Mavromatos, Phys. Rev. D 100, 096005 (2019)

25. F. Blaschke, P. Benes, Prog. Theor. Exp. Phys. 073B03 (2018)

26. Y.M. Cho, Phys. Rev. D 21, 1080 (1980)

27. Y.M. Cho, Phys. Rev. Lett. 46, 302 (1981)

28. Y.M. Cho, Phys. Rev. D 23, 2415 (1981)

29. W.S. Bae, Y.M. Cho, S.W. Kimm, Phys. Rev. D 65, 025005 (2002)

30. L. Faddeev, A. Niemi, Phys. Rev. Lett. 82, 1624 (1999)

31. L. Faddeev, A. Niemi, Phys. Lett. B 449, 214 (1999)

32. S. Shabanov, Phys. Lett. B 458, 322 (1999)

33. S. Shabanov, Phys. Lett. B 463, 263 (1999)

34. H. Gies, Phys. Rev. D 63, 125023 (2001)

35. R. Zucchini, Int. J. Geom. Meth. Mod. Phys. 1, 813 (2004)

36. K. Kondo, S. Kato, A. Shibata, T. Shinohara, Phys. Rep. 579, 1 (2015)

37. S. Coleman, Aspects of Symmetry (Cambridge Univ. Press, Cambridge, 1985)

38. T. Vachaspati, M. Barriola, Phys. Rev. Lett. 69, 1867 (1992)

39. T. Vachaspati, M. Barriola, Phys. Rev. Lett. 69, 1867 (1992)

40. M. Barriola, T. Vachaspati, M. Bucher, Phys. Rev. D 50, 2819 (1994)

41. B. Cabrera, Phys. Rev. Lett. 48, 1378 (1982)

42. A. Abrokosov, JETP (Sov. Phys.) 5, 1173 (1957)

43. H. Nielsen, P. Olesen, Nucl. Phys. B 61, 45 (1973) 